\title{
SQL QUERY EXPRESSED IN RELATIONAL CALCULUS
}

\author{
HO THUAN, TRAN THIEN THANH
}

\begin{abstract}
In this paper, we present some results on the equivalence between SQL queries and relation calculus expressions. These results are used to translate SQL queries into equivalent expressions of relational algebra.
\end{abstract}

\section{INTRODUCTION}

Stefano Ceri and Georg Gottlob, in [1] presented a translator from a subset of SQL queries into relational algebra. We have extended the results in [1] for subqueries with GROUP BY clause which can be nested at any level of a SQL query, and some extensions to condition of HAVING clause. This work is a basis step for this translation. In this paper, we have proved some results on the equivalence between SQL query and relational calculus expression.

\section{BASIS KNOWLEDGE}

\section{Relational calculus}

A relational calculus expression is of the form

where

$$
\{t(\langle\text { components }\rangle) \mid \psi(t)\},
$$

- $t$ is a tuple variable;

- components is a list of components of the form:

$+A_{i}$ - is an attribute,

$+R . A_{i}-R$ is a relation name, $A_{i}$ is an attribute of $R$;

- $\psi(t)$ is a formula building from the atoms and collection of logical operators.

In order to use aggregate function in relational calculus, we extended the components to accept the form $F_{i}\left[A_{j}\right]$, where $F_{i}$ is a function and $A_{j}$ is an attribute, and other extensions to the atoms of formula $\psi$ of some types such that: $F_{i}\left[A_{j}\right](s) \Theta u\left[A_{i}\right], F_{i}\left[A_{j}\right](s) \Theta a$, where $F_{i}\left[A_{j}\right](s)$ is value of function $F_{i}$ computed on attribute $A_{j}$ for the tuple $s, a$ is a constant, and $\Theta \in\{=, \neq,>, \geq,<, \leq\}$.

\section{Structured Query Language (SQL)}

a. Syntax of $S Q L$ query

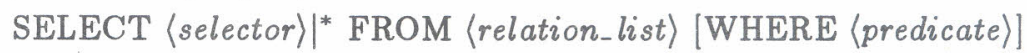

[GROUP BY $\left\langle g b_{-} a t t r\right\rangle$ [HAVING $\langle$ hav_condition $\left.\left.\rangle\right]\right]$

b. The meaning of clauses

The SELECT clause indicates attributes and functions are selected. The asterisk denote for all attributes of $\langle$ relation $-l i s t\rangle$.

The FROM clause indicates relations used for query.

Note: Every SQL query must have at least the SELECT clause and the FROM clause.

The WHERE clause indicates condition used to select tuples, only select tuples that satisfying the condition.

The GROUP BY clause indicates attributes, those used to group the tuples. 
The HAVING clause indicates condition used to select groups, only select groups satisfying the condition.

c. The operators

The operators used to combining results of SQL queries: INTERSECT, UNION, MINUS.

\section{Notation and relations used to illustrate}

\section{$\square$ Notation}

- car(list of relational expressions) indicates the Cartesian product of all the relational expressions.

- $\operatorname{attr}$ (list of relations) is the set of the attributes in the attributes schema of the specified relations.

- attr(relational expressions) is the set of attributes occurring in the results produced by the evaluation of a relational expression.

- rels(list of attributes) is the set of relations having the specified attributes.

- rels(relational expression) is the set of the relations, whose attributes appear in the relational expression.

- extrattr(predicate) is the set of attributes which appear in the predicate.

- extrels (predicate) is the set of relations whose attributes appear in the predicate.

- meaning $(Q)$ is the relation results of query $Q$.

$\square$ The relations used to illustrate

To illustrate, we use the database relations in Date's book [2].

- Relations $S$ - Suppliers

\begin{tabular}{|l|l|l|l|}
\hline SCODE & SNAME & STATUS & CITY \\
\hline S1 & Smith & 20 & London \\
S2 & Jones & 10 & Paris \\
S3 & Blake & 30 & Paris \\
\hline
\end{tabular}

- Relation P - Products

\begin{tabular}{|l|l|l|l|l|}
\hline PCODE & PNAME & COLOR & WEIGHT & CITY \\
\hline P1 & Nut & Red & 12 & Lodon \\
P2 & Bolt & Green & 17 & Paris \\
P3 & Screw & Blue & 17 & Rome \\
P4 & Screw & Red & 14 & London \\
\hline
\end{tabular}

- Relation SP - Supplier-Product

\begin{tabular}{|l|l|l|}
\hline SCODE & PCODE & QTY \\
\hline S1 & P1 & 300 \\
S1 & P2 & 200 \\
S1 & P3 & 400 \\
S2 & P1 & 300 \\
S2 & P2 & 400 \\
S3 & P2 & 400 \\
\hline
\end{tabular}

\section{SQL QUERIES EXPRESSED IN RELATIONAL CALCULUS}

1. Notation and definition

Let $Q$ be a SQL query of the form: 


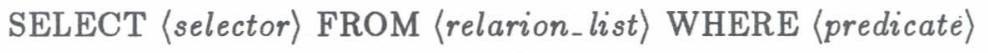

GROUP BY $\left\langle g b_{-} a t t r\right\rangle$ HAVING 〈hav_condition $\rangle$

- $Q$. 〈component $\rangle$ denotes the corresponding components of $Q$,

- $Q .\langle$ component_1 1$\rangle \cdot\langle$ component_ 2$\rangle$ denotes the corresponding component of $Q .\langle$ component_ 1$\rangle$,

- $Q .\langle$ selector $\rangle \cdot\langle$ attrs $\rangle$ is list of attributes in $Q .\langle$ selector $\rangle$,

- $Q .\langle$ selector $\rangle \cdot\langle$ function_list $\rangle$ is list of functions in $Q .\langle$ selector $\rangle$.

In this paper we assume $Q .\langle$ selector $\rangle .\langle$ function_list $\rangle=\left\{F_{i}\left[A_{j}\right]\right\}, i=1, \ldots, k, F_{i}\left[A_{j}\right]$ is a function $F_{i}$ computed on attribute $A_{j}$.

Example: Let $R$ be relation, $R$ has the schema $R(A, B) . Q$ be a SQL query of the form SELECT R.A, R.B, $\operatorname{SUM}(R . A)$ FROM $R$, then
$Q .\langle$ selector $\rangle=\{R . A, R . B, \operatorname{SUM}(R . A)\}$
$Q .\langle$ selector $\rangle \cdot\langle$ attrs $\rangle=\{R . A, R: B\}$
$Q .\langle$ selector $\rangle \cdot\langle$ function_list $\rangle=\{\operatorname{SUM}(R . A)\}$

- $F_{i}\left[R ; U ; A_{j}\right](t)$ (where $R$ is a relation, $U$ is a subset of $\operatorname{attr}(R), A_{j}$ is a attribute of $R, A_{j} \notin U$, $t$ is a tuple in $R$ ): is the value of function $F_{i}$ computed on attribute $A_{j}$ of relation $R$ with group-attribute $U$ for tuple $t$ of $R$.

Note: The values of $F_{i}\left[A_{j}\right]$ are same with tuples in a group (Fig. 1).

$\forall t, t^{\prime} \in R$, if $t^{\prime}(U)=t(U)$ then $F_{i}\left[R ; U ; A_{j}\right]\left(t^{\prime}\right)=F_{i}\left[R ; U ; A_{j}\right](t)$

\begin{tabular}{|l|l|c|}
\hline$\cdots$ & $U$ & $F_{i}\left[R ; U ; A_{j}\right]$ \\
\hline$r_{1}$ & $g_{1}$ & $f_{1}$ \\
$r_{2}$ & $g_{1}$ & $f_{1}$ \\
\hline$r_{3}$ & $g_{2}$ & $f_{2}$ \\
$r_{4}$ & $g_{2}$ & $f_{2}$ \\
$r_{5}$ & $g_{2}$ & $f_{2}$ \\
\hline
\end{tabular}

Fig. 1

Definition 1. Let $Q$ be a SQL query and $E$ be a relational calculus expression, we say that $Q$ is equivalent to $E$ iff the results of $Q$ and $E$ are the same when we substitute the same relations for identical name in the two expressions.

When $Q$ is equivalent to $E$, we say that $E$ is $Q$ expressed in relational calculus and denoted $Q=E$.

Definition 2. Let $Q, Q^{\prime}$ be SQL queries. We say that $Q$ is equivalent to $Q^{\prime}$, denoted $Q=Q^{\prime}$, iff when we substitute the same relations for identical name in the two expressions, we get the same result.

We have $Q=Q^{\prime} \Leftrightarrow$ meaning $(Q)=$ meaning $\left(Q^{\prime}\right)$.

2. The top level query

Let $Q$ be a SQL query of the form:

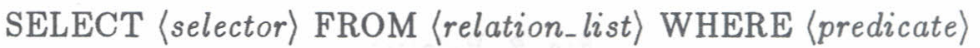

GROUP BY $\left\langle g b_{-} a t t r\right\rangle$ HAVING 〈hav_condition〉

Based on the meaning of $Q$, we have:

$$
\begin{aligned}
& Q=\left\{t(Q \cdot\langle\text { selector }\rangle) \mid \exists t^{\prime}\left(R\left(t^{\prime}\right) \wedge t(Q \cdot\langle\text { selector }\rangle \cdot\langle\text { attrs }\rangle)=\right.\right. \\
& t^{\prime}(Q \cdot\langle\text { selector }\rangle \cdot\langle\text { attr }\rangle) \wedge F\left(t^{\prime}\right) \wedge \forall r(R(r) \wedge(r(Q \cdot\langle\text { gb_attr }\rangle)= \\
&\left.\left.\left.t^{\prime}\left(Q \cdot\left\langle g b_{-} \text {attr }\right\rangle\right) \rightarrow H(r)\right)\right) \wedge\left(t\left(F_{i}\left[A_{j}\right]\right)=F_{i}\left[R^{\prime \prime} ; Q \cdot\left\langle g b_{-} \text {attr }\right\rangle ; A_{j}\right]\left(t^{\prime}\right) i=1, \ldots, k\right)\right\}
\end{aligned}
$$

where 
$R=\operatorname{car}(Q \cdot\langle$ relation-list $\rangle)$,

$F(t)$ is predicate $Q \cdot\langle$ predicate $\rangle$,

$H(t)$ is condition $Q .\left\langle h a v_{-}\right.$condition $\rangle$,

$R^{\prime}=\{t \mid R(t) \wedge F(t)\}$ - set of tuples of $R$, those are satisfying $Q$. (predicate〉,

$R^{\prime \prime}=\left\{t \mid R^{\prime}(t) \wedge \forall t^{\prime}\left(R^{\prime}\left(t^{\prime}\right) \wedge\left(t^{\prime}\left(Q \cdot\left\langle g b_{-} a t t r\right\rangle\right)=t\left(Q \cdot\left\langle g b_{-} a t t r\right\rangle\right) \rightarrow H\left(t^{\prime}\right)\right)\right)\right\}$.

We rewrite equation (1):

$$
\begin{aligned}
& Q=\left\{t(Q .\langle\text { selector }\rangle) \mid \exists t^{\prime}\left(R^{\prime \prime}\left(t^{\prime}\right) \wedge t(Q \cdot\{\text { selector }\rangle \cdot\langle\text { attrs }\rangle)=\right.\right. \\
&\left.t^{\prime}(Q \cdot\langle\text { selector }\rangle \cdot\langle\text { attrs }\rangle) \wedge\left(t\left(F_{i}\left[A_{j}\right]\right)=F_{i}\left[R^{\prime \prime} ; Q \cdot\langle\text { gb_attr }\rangle ; A_{j}\right]\left(t^{\prime}\right) i=1, \ldots, k\right)\right\}
\end{aligned}
$$

\section{The subquery}

The subqueries may be used in conjunction with the IN, ALL, EXISTS, ... operators.

Example. Find the name of suppliers, those do not supply product P1.

$Q=$ SELECT SNAME FROM S WHERE "P1" NOT IN

(SELECT PCODE FROM SP WHERE SCODE=S.SCODE)

We consider the subquery:

$Q^{\prime}=$ SELECT PCODE FROM SP WHERE SCODE=S.SCODE

Remark.

1) The subquery $Q^{\prime}$ is dependent on S.SCODE of $S$.

2) To have meaning $(Q)$, we need to have meaning $\left(Q^{\prime}\right)$.

Definition 3 (External relation, External attribute of subquery).

Let $Q$ be a subquery, $R$ be a relation, if there exists attribute(s) of $R$ appear in $Q$, but $R$ not in $Q$. $\langle$ relation_list $\rangle$ then $R$ is called external relation of $Q$.

Every attribute of $Q^{\prime}$ s external relation is called external attribute of $Q$.

The set of all external attributes of $Q$ is denoted by $\operatorname{Other}(Q)$.

Definition 4 (The relation result of subquery).

Let $Q$ be a subquery, $\operatorname{Other}(Q)$ is set of all external attributes of $Q . S=\operatorname{car}(\operatorname{rels}(\operatorname{other}(Q)))$.

For each $s \in S, Q(s)$ is a subquery, it is obtained by replace each attribute $A_{i} \in \operatorname{Other}(Q)$ by $s\left(A_{i}\right)$. Let $R_{s}=$ meaning $(Q(s))$.

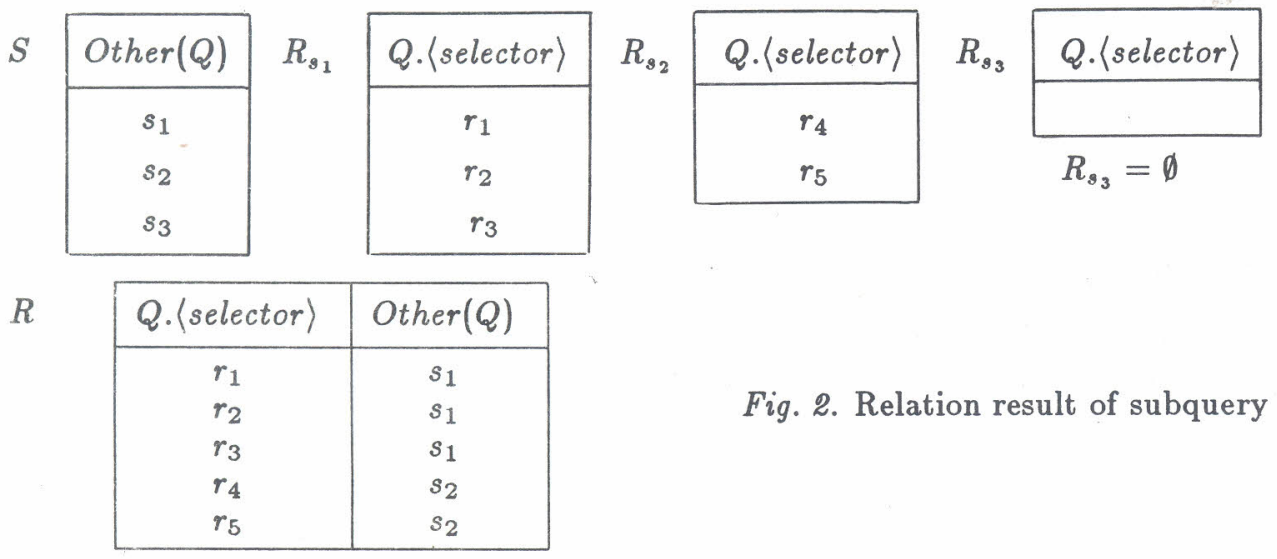

Definition: The relation result of subquery $Q$ is defined by expression:

$$
\left\{(r, s) \mid S(s) \wedge R_{s}(r)\right\}
$$


Example: Let $Q$ be a subquery

SELECT PCODE FROM SP WHERE SCODE=S.SCODE

Other $(Q)=\{$ SCODE, SNAME, STATUS, CITY $\}$

$$
\begin{aligned}
& s_{1}=(S 1, \text { Smith }, 20, \text { London }) \\
& s_{2}=(S 2, \text { Lones, } 10, \text { Paris }) \\
& s_{3}=(S 3, \text { Blake, } 30, \text { Paris })
\end{aligned}
$$

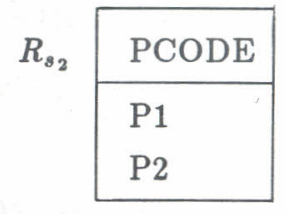

The relation result of subquery $Q$

$R$

\begin{tabular}{|l|l|l|l|l|}
\hline PCODE & S.SCODE & SNAME & STATUS & CITY \\
\hline P1 & S1 & Smith & 20 & London \\
P2 & S1 & Smith & 20 & London \\
P3 & S1 & Smith & 20 & London \\
\hline P1 & S2 & Jones & 10 & Paris \\
P2 & S2 & Jones & 10 & Paris \\
\hline P2 & S3 & Blake & 30 & Paris \\
\hline
\end{tabular}

By equation (1), we have:

$$
\begin{aligned}
R_{s}=\left\{t(Q .\langle\text { selector }\rangle) \mid \exists t^{\prime}\left(R_{s}^{*}\left(t^{\prime}\right) \wedge t(Q .\langle\text { selector }\rangle \cdot\langle\text { attrs }\rangle)=\right.\right. \\
\left.\left.t^{\prime}(Q \cdot\langle\text { selector }\rangle \cdot\langle\text { attrs }\rangle) \wedge\left(t\left(F_{i}\left[A_{j}\right]\right)=F_{i}\left[R_{s}^{*} ; Q .\langle\text { gb_attr }\rangle ; A_{j}\right]\left(t^{\prime}\right) i=1, \ldots, k\right)\right)\right\},
\end{aligned}
$$

where

$$
R_{s}^{*}=\left\{r \mid R(r) \wedge F(r, s) \wedge \forall r^{\prime}\left(R\left(r^{\prime}\right) \wedge F\left(r^{\prime}, s\right) \wedge\left(r\left(Q \cdot\left\langle g b_{-} a t t r\right\rangle\right)=r^{\prime}\left(Q \cdot\left\langle g b_{-} a t t r\right\rangle\right) \rightarrow H\left(r^{\prime}, s\right)\right)\right)\right\}
$$

\section{RESULTS}

Theorem 1. Let $Q$ be a subquery of the form

SELECT $\langle$ selector $\rangle$ FROM $\langle$ relation_list $\rangle$ WHERE $\langle$ predicate $\rangle$ GROUP BY $\left\langle g b_{-} a t t r\right\rangle$

HAVING 〈hav_condition $\rangle$ then

$$
\begin{aligned}
Q=\{ & t(Q \cdot\langle\text { selector }\rangle \cup \operatorname{Other}(Q)) \mid \exists\left(P_{F H}(p) \wedge t(Q .\langle\text { selector }\rangle \cdot\langle\text { attrs }\rangle \cup \text { Other }(Q))=\right. \\
& p(Q .\langle\text { selector }\rangle \cdot\langle\text { attrs }\rangle \cup \text { Other }(Q)) \wedge \\
& \left.\left.\left(t\left(F_{i}\left[A_{j}\right]\right)=F_{i}\left[P_{F H} ; Q .\left\langle g b_{-} \text {attr }\right\rangle \cup \operatorname{Other}(Q) ; A_{j}\right](p) i=1, \ldots, k\right)\right)\right\}
\end{aligned}
$$

where

$$
\begin{aligned}
& P=\operatorname{car}(Q \cdot\langle\text { relation_list }\rangle \cup \operatorname{rels}(\text { Other }(Q))), \\
& P_{F}=\{p \mid P(p) \wedge F(p)\}, \\
& P_{F H}= \\
& \left\{p \mid P_{F}(p) \wedge \forall p^{\prime}\left(P_{F}\left(p^{\prime}\right) \wedge p^{\prime}\left(Q \cdot\left\langle g b_{\text {_attr }}\right\rangle \cup \operatorname{Other}(Q)\right)=p\left(Q \cdot\left\langle g b_{-} \text {attr }\right\rangle \cup \text { Other }(Q)\right) \rightarrow H\left(p^{\prime}\right)\right)\right\} .
\end{aligned}
$$

Define $P^{*}=\left\{(t, s) \mid S(s) \wedge R_{s}^{*}(t)\right\}$. The proof of Theorem 1 is based on Lemma 1, and Lemma 2 . We omit here the proof of these lemmas. 
Lemma 1. We have $P^{*}=P_{F H}$.

Lemma 2. For every $p \in P^{*}, p=(t, s)$, where $s \in S, t \in R_{s}^{*}$. Let $F_{i}$ be a function, $A_{j} \in$ $\operatorname{attr}(Q \cdot\langle$ relation_list $\rangle)$ we have:

$$
F_{i}\left[R_{s}^{*} ; Q \cdot\left\langle g b_{-} a t t r\right\rangle ; A_{j}\right](t)=F_{i}\left[P^{*} ; Q \cdot\left\langle g b_{-} a t t r\right\rangle \cup \operatorname{Other}(Q) ; A_{j}\right](p)
$$

Proof of Theorem 1. By equation (3) we have the equivalence of $Q$ with the expression $E$ defined by:

$$
\begin{aligned}
E=\{ & \left.t(Q \cdot\langle\text { selector }\rangle \cup \operatorname{Other}(Q)) \mid \exists s\left(S(s) \wedge t(\operatorname{Other}(Q))=s \wedge \exists r\left(R_{s}(r) \wedge t(Q \cdot\langle\text { selector }\rangle)=r\right)\right)\right\} \\
& =\left\{(r, s) \mid S(s) \wedge R_{s}(r)\right\}
\end{aligned}
$$

where

$$
\begin{aligned}
R_{s}=\left\{t(Q \cdot\langle\text { selector }\rangle) \mid \exists t^{\prime}\left(R_{s}^{*}\left(t^{\prime}\right) \wedge t(Q \cdot\langle\text { selector }\rangle \cdot\langle\text { attrs }\rangle)\right)=\right. \\
\left.t^{\prime}(Q \cdot\langle\text { selector }\rangle \cdot\langle\text { attrs }\rangle) \wedge\left(t\left(F_{i}\left[A_{j}\right]=F_{i}\left[R_{s}^{*} ; Q \cdot\langle\text { gb_attr }\rangle ; A_{j}\right]\left(t^{\prime}\right) i=1, \ldots, k\right)\right)\right\} .
\end{aligned}
$$

Let

$$
\begin{aligned}
& E^{\prime}=\left\{t(Q .\langle\text { selector }\rangle \cup \operatorname{Other}(Q)) \mid \exists p\left(P_{F H}(p) \wedge\right.\right. \\
& t(Q .\langle\text { selector }\rangle \cdot\langle\text { attrs }\rangle \cup \operatorname{Other}(Q))=p(Q \cdot\langle\text { selector }\rangle \cdot\langle\text { attrs }\rangle \cup \operatorname{Other}(Q)) \wedge \\
& \left.\left.\left(t\left(F_{i}\left[A_{j}\right]\right)=F_{i}\left[P_{F H} ; Q \cdot\left\langle g b_{-} \text {attr }\right\rangle \cup \operatorname{Other}(Q) ; A_{j}\right](p) i=1, \ldots, k\right)\right)\right\} .
\end{aligned}
$$

By Lemma 1, we have

$$
\begin{aligned}
& E^{\prime}=\left\{t(Q \cdot\langle\text { selector }\rangle \cup \text { Other }(Q)) \mid \exists p\left(P^{*}(p) \wedge\right.\right. \\
& t(Q \cdot\langle\text { selector }\rangle \cdot\langle\text { attrs }\rangle \cup \operatorname{Other}(Q))=p(Q \cdot\langle\text { selector }\rangle \cdot\langle\text { attrs }\rangle \cup \operatorname{Other}(Q)) \wedge \\
& \left.\left.\left(t\left(F_{i}\left[A_{j}\right]\right)=F_{i}\left[P^{*} ; Q \cdot\langle\text { gb_attr }\rangle \cup \operatorname{Other}(Q) ; A_{j}\right](p) i=1, \ldots, k\right)\right)\right\} .
\end{aligned}
$$

We show that $E=E^{\prime}$

a) First we show that $E^{\prime} \subseteq E$

$\forall t \in E^{\prime} \Rightarrow \exists p \in P^{*}$.

$t(Q .\langle$ selector $\rangle \cdot\langle$ attrs $\rangle \cup \operatorname{Other}(Q))=p(Q .\langle$ selector $\rangle .\langle$ attrs $\rangle \cup \operatorname{Other}(Q))$

$t\left(F_{i}\left[A_{j}\right]\right)=F_{i}\left[P^{*} ; Q \cdot\left\langle g b_{-}\right.\right.$attr $\left.\rangle \cup \operatorname{Other}(Q) ; A_{j}\right](p) i=1, \ldots, k$.

Since $p \in P^{*}$ then $p=(r, s)$ where $s \in S, r \in R_{s}^{*}$.

We have $t(\operatorname{Other}(Q))=p(\operatorname{Other}(Q))=s \in S$

$$
\begin{aligned}
& t(Q .\langle\text { selector }\rangle \cdot\langle\text { attrs }\rangle)=p(Q \cdot\langle\text { selector }\rangle \cdot\langle\text { attrs }\rangle)=r(Q .\langle\text { selector }\rangle \cdot\langle\text { attrs }\rangle) \\
& t\left(F_{i}\left[A_{j}\right]\right)=F_{i}\left[P^{*} ; Q \cdot\left\langle g b_{-} \text {attr }\right\rangle \cup \operatorname{Other}(Q) ; \dot{A}_{j}\right](p) \forall i=1, \ldots, k .
\end{aligned}
$$

By Lemma 2, we have $t\left(F_{i}\left[A_{j}\right]\right)=F_{i}\left[R_{s}^{*} ; Q .\left\langle g b_{-} a t t r\right\rangle ; A_{j}\right](t) \forall i=1, \ldots, k$.

Let $t^{\prime}=t(Q \cdot\langle$ selector $\rangle)$ then

$$
t^{\prime}(Q \cdot\langle\text { selector }\rangle \cdot\langle\text { attrs }\rangle)=t^{\prime}(Q \cdot\langle\text { selector }\rangle \cdot\langle\text { attrs }\rangle)=r(Q \cdot\langle\text { selector }\rangle \cdot\langle\text { attrs }\rangle)
$$

and $t^{\prime}\left(F_{i}\left[A_{j}\right]\right)=F_{i}\left[P^{*} ; Q \cdot\left\langle g b_{-}\right.\right.$attr $\left.\rangle \cup \operatorname{Other}(Q) ; A_{j}\right](p)=F_{i}\left[R_{s}^{*} ; Q\left\langle g b_{-} a t t r\right\rangle ; A_{j}\right](r) \forall i=1, \ldots, k$ so $t^{\prime} \in R_{s}$.

Clearly $t=\left(t^{\prime}, s\right) \Rightarrow t \in E s o E^{\prime} \subseteq E(*)$

b) Now we have to show that $E \subseteq E^{\prime}$

$\forall t \in E \Rightarrow t=(r, s)$ where $s \in S, r \in R_{s}$. 
Since $r \in R_{s}$ then $\exists t^{\prime} \in R_{s}^{*}, r(Q \cdot\langle$ selector $\rangle \cdot\langle$ attrs $\rangle)=t^{\prime}(Q .\langle$ selector $\rangle \cdot\langle$ attrs $\rangle)$ and $r\left(F_{i}\left[A_{j}\right]\right)=F_{i}\left[R_{s}^{*} ; Q \cdot\left\langle g b_{-} a t t r\right\rangle ; A_{j}\right]\left(t^{\prime}\right) \forall i=1, \ldots, k$.

Let $p=\left(t^{\prime}, s\right)$, we have $p \in P^{*}$ and

$$
\begin{aligned}
& t(Q \cdot\langle\text { selector }\rangle \cdot\langle\text { attrs }\rangle)=r(Q \cdot\langle\text { selector }\rangle \cdot\langle\text { attrs }\rangle)=t^{\prime}(Q .\langle\text { selector }\rangle \cdot\langle\text { attrs }\rangle) \\
& =p(Q \cdot\langle\text { selector }\rangle \cdot\langle\text { attrs }\rangle) \\
& t\left(F_{i}\left[A_{j}\right]\right)=r\left(F_{i}\left[A_{j}\right]\right)=F_{i}\left[R_{s}^{*} ; Q .\left\langle g b_{-} \text {attr }\right\rangle ; A_{j}\right]\left(t^{\prime}\right) \forall i=1, \ldots, k
\end{aligned}
$$

By Lemma 2, we have

$$
t\left(F_{i}\left[A_{j}\right]\right)=F_{i}\left[P^{*} ; Q\left\langle g b_{-} a t t r\right\rangle \cup \operatorname{Other}(Q) ; A_{j}\right](p) \forall i=1, \ldots, k \text { so } t \in E^{\prime} \Rightarrow E^{\prime} \subseteq E \quad(* *)
$$

From $\left({ }^{*}\right)$ and $\left({ }^{* *}\right) \Rightarrow E=E^{\prime}$

By Theorem 1, we have the following remark:

Remark 1. Let $Q$ be a query of the form

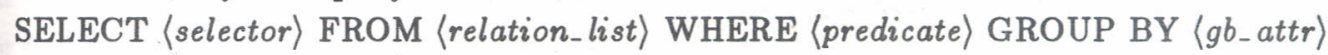

HAVING 〈hav_condition〉

then $Q$ is equivalent to the query $Q^{\prime}$ of the form

SELECT $Q .\langle$ selector $\rangle \cup \operatorname{Other}(Q)$ FROM $Q .\langle$ relation_list $\rangle \cup$ rels $($ Other $(Q))$

WHERE $Q .\langle$ predicate $\rangle$ GROUP BY $Q .\left\langle g b_{-}\right.$attr $\rangle \cup$ Other $(Q)$ HAVING $Q$. $\langle$ hav_condition $\rangle$.

Theorem 2. Let $Q$ be a query of the form

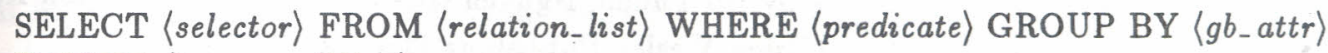

HAVING 〈hav_condition〉

then we have

$$
\begin{aligned}
Q=\{ & t(Q .\langle\text { selector }\rangle \cup \operatorname{Other}(Q)) \mid \exists r\left(R^{*}(r) \wedge t(Q .\langle\text { selector }\rangle \cdot\langle\text { attrs }\rangle \cup \operatorname{Other}(Q))=\right. \\
& r(Q .\langle\text { selector }\rangle \cdot\langle\text { attrs }\rangle \cup \operatorname{Other}(Q)) \wedge \\
& \left.\left.\left(t\left(F_{i}\left[A_{j}\right]\right)=F_{i}\left[R^{*} ; Q .\left\langle g b_{-} \text {attr }\right\rangle \cup \operatorname{Other}(Q) ; A_{j}\right](r) i=1, \ldots, k\right)\right)\right\}
\end{aligned}
$$

where $Q .\left\langle n g b_{-} q u e r y\right\rangle^{*}$ denotes the query of the form

SELECT * FROM $Q .\langle$ relation_list $\rangle$ WHERE $Q \cdot\langle$ predicate $\rangle$

$R=\operatorname{car}\left(\right.$ meaning $\left(Q .\left\langle n g b_{-} q u e r y\right\rangle^{*}\right) \cup\left(\operatorname{rels}(\operatorname{Other}(Q))-\operatorname{rels}\left(\right.\right.$ meaning $\left.\left(Q .\left\langle n g b_{-} q u e r y\right\rangle^{*}\right)\right)$

$R^{*}=\left\{r \mid R(r) \wedge \forall r^{\prime}\left(R\left(r^{\prime}\right) \wedge\left(r^{\prime}\left(Q .\left\langle g b_{\text {a attr }}\right\rangle \cup \operatorname{Other}(Q)\right)=r\left(Q \cdot\left\langle g b_{\text {_attr }}\right\rangle \cup \operatorname{Other}(Q)\right) \rightarrow H\left(r^{\prime}\right)\right)\right)\right\}$

Theorem 2 allows to express queries with GROUP BY clause by the result of queries without GROUP BY clause. 3.

The proof of Theorem 2 is based on the Lemma 3. As above, we omit here the proof of Lemma

Lemma 3. We have $R=\{p \mid P(p) \wedge F(p)\}$.

\section{Proof of Theorem 2.}

By Lemma 3 we have $R^{*}=P_{F H}$.

By Theorem 1 clearly

$$
\begin{aligned}
Q=\{ & t(Q \cdot\langle\text { selector }\rangle \cup \operatorname{Other}(Q)) \mid \exists r\left(R^{*}(r) \wedge\right. \\
& t(Q \cdot\langle\text { selector }\rangle \cdot\langle\text { attrs }\rangle \cup \operatorname{Other}(Q))=r(Q \cdot\langle\text { selector }\rangle \cdot\langle\text { attrs }\rangle \cup \operatorname{Other}(Q)) \wedge \\
& \left.\left.\left(t\left(F_{i}\left[A_{j}\right]\right)=F_{i}\left[R^{*} ; Q \cdot\left\langle g b_{-} \text {attr }\right\rangle \cup \operatorname{Other}(Q) ; A_{j}\right](r) i=1, \ldots, k\right)\right)\right\} . \quad \square
\end{aligned}
$$

By Theorem 2, we have the following remark: 
Remark 2. Let $Q$ be a query of the form

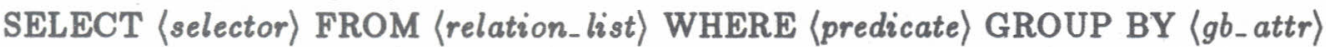

HAVING 〈hav_condition〉

then the following queries are equivalent:

i) $Q$

ii) $Q^{\prime}=$ SELECT $Q \cdot\langle$ selector $\rangle \cup$ Other $(Q)$ FROM TEMP1 GROUP BY $Q \cdot\left\langle g b_{-} a t t r\right\rangle \cup O t h e r(Q)$, where TEMP1 $=$ meaning $\left(Q^{*}\right)$

with $Q^{*}=$ SELECT ${ }^{*}$ FROM $Q .\langle$ relation_list $\rangle$ WHERE $Q \cdot\langle$ predicate $\rangle$ GROUP. BY $Q .\left\langle g b_{-} a t t r\right\rangle$ HAVING $Q \cdot\left\langle h a v_{-}\right.$condition $\rangle$.

iii) $Q^{\prime \prime}=\operatorname{SELECT~} Q .\langle$ selector $\rangle \cup \operatorname{Other}\left(Q \cdot\left\langle n g b_{-}\right.\right.$query $\left.\rangle\right)$FROM TEMP2 GROUP BY $Q .\left\langle g b_{-} a t t r\right\rangle$ UOther $(Q .\langle n g b$ - query $\rangle)$ HAVING $Q$. 〈hav_condition $\rangle$,

where TEMP2 $=$ meaning $\left(Q \cdot\left\langle n g b_{-} \text {query }\right\rangle^{*}\right)$.

\section{REFERENCES}

[1] S. Ceri, G. Gottlob, Translating SQL into Relational Algebra: Optimization, Semantics, and Equivalence of SQL Queries, IEEE Trans. Comput., Vol. SE-11, No. 4 (1985) 324-345.

[2] C. J. Date., An introduction to Database System, 2nd ed., Addision-Wesley Publishing Compar: 1997. (The translation to Vietnamese by Ho Thuan, Nguyen Quang Vinh, Nguyen Xuan Huy).

[3] R. F. Lans., Introduction to $S Q L$, Addision-Wesley Publishing Company, 1988.

[4] J. Paredaens, P.D. Bra, M. Gyssen, D.V. Gucht., The structure of the Relational Database Model, Springer Verlag, 1989.

[5] J. Ulman, Principles of Database Systems, Computer Science Press, 1980.

Tơm tăt. Trong bài báo này chúng tôi trình bày một số kết quá về sự tương đương giữa nhữag câu hỏi của SQL và biều thức trong phép tính quan hệ, một số tính chất của câu hỏi trong şir. Những kết quả này được sử dụng cho việc chuyển dịch câu hỏi của SQL vào đại số quan hệ.

(1) Institute of Information Technology.

Received: March 11, 1998

(2) Pedagogical Institute of Quy Nhon. 